\title{
El Instituto de Derechos Humanos "Gregorio Peces-Barba" sigue un año más trabajando en la defensa de los derechos
}

El pasado 15 de septiembre se inauguró un nuevo curso en el Instituto de Derechos Humanos "Gregorio Peces-Barba" ${ }^{1}$ con la Conferencia de María José Añón, Catedrática de Filosofía del Derecho e investigadora del Institut de Drets Humans de la Universitat de València, 'Medidas de austeridad: una evaluación desde los derechos humanos' a la que asistieron estudiantes de los programas del Instituto ya en marcha: Máster Universitario en Estudios Avanzados en Derechos Humanos, Doctorado en Estudios Avanzados en Derechos Humanos, Máster en Derechos Fundamentales y Máster online en Derechos Fundamentales.

Tras esta inauguración, el Instituto ha seguido con su trabajo habitual, aunque debido a la situación provocada por la COVID-19, éste ha tenido que desarrollarse en formato online síncrono, con excepción de algunas actividades docentes en las que ha sido posible la presencialidad.

En estos meses se han organizado diferentes Talleres y celebrado diversas jornadas, seminarios y encuentros, algunos de los cuáles han tenido como eje el análisis de los efectos de la pandemia desde una perspectiva de derechos.

Entre todas estas actividades, pueden destacarse la Jornada 'El tiempo de los derechos' del 17 de noviembre, que incluyó una primera sesión en recuerdo del profesor de la Universidad de Zaragoza e integrante de la Red Tiempo de los derechos, Manuel Calvo, y la Jornada celebrada el 10 de diciembre, el día de los derechos humanos, en la que se conmemoró el 30 aniversario del Instituto y que giró en torno a la aportación de Gregorio Peces-Barba al estudio de los derechos. Esta jornada se aprovechó también para anunciar oficialmente el cambio de denominación del Instituto de Derechos Humanos Bartolomé de las Casas a Instituto de Derechos Humanos “Gregorio Peces-

1 El 10 de diciembre de 2020 se anunció oficialmente el cambio de denominación del Instituto de Derechos Humanos "Bartolomé de las Casas" a Instituto de Derechos Humanos "Gregorio Peces-Barba". 
Barba" en homenaje a su fundador y referente en la investigación y docencia de los derechos humanos.

Por otro lado, la Asociación de Estudiantes y Egresados/as del IDHBC ha seguido sumando nuevas adhesiones. El 24 de septiembre, se presentó la delegación argentina en una jornada online en la que se reflexionó sobre "Derechos humanos, vulnerabilidades y pandemia".

Además, el Instituto ha puesto en marcha una nueva convocatoria de la Clínica Jurídica Javier Romañach dirigida a estudiantes de Grado y Posgrado de la UC3M que quieran contribuir a mejorar la situación de las personas con discapacidad y aprender derechos humanos de una forma distinta, comprometida e integral. En este curso, estudiantes y profesorado están trabajando en programas sobre los ODS, la Agenda 2030 y la Convención sobre los Derechos de las Personas con Discapacidad (CDPD), la condición europea de persona con discapacidad, el deporte inclusivo y federaciones deportivas, la discriminación de las personas con autismo y la reforma de la capacidad a la luz de la CDPD en colaboración con CERMI Estatal, Autismo España y SOLCOM.

Una de las principales novedades de este curso es que el Instituto ha lanzado junto con Tirant Formación el Máster en Filosofía Jurídica y Política Contemporánea (online) destinado a personas que quieran iniciarse en el estudio y la investigación sobre el Derecho en general, y sobre la Filosofía del Derecho en particular, completar tu formación en esta materia o actualizarse en las cuestiones más importantes que afectan al Derecho y a su relación con la Ética y la Política. Se trata de un programa online especializado e interdisciplinar orientado hacia el estudio de la filosofía jurídica y política contemporánea, impartido por importantes especialistas en la materia y que cuenta con un material bibliográfico y docente que destaca por su actualidad y solvencia. El Máster comenzará en el mes de febrero y las y los primeros estudiantes matriculados ya asistieron a la Conferencia inaugural del curso.

Lara Lucía Cuenca Gómez

DERECHOS Y LIBERTADES

ISSN: $1133-0937$

Número 44, Época II, enero 2021, pp. 391-394 